\title{
Electric solar wind sail mass budget model
}

\author{
P. Janhunen ${ }^{1}$, A. A. Quarta ${ }^{2}$, and G. Mengali ${ }^{2}$ \\ ${ }^{1}$ Finnish Meteorological Institute, Helsinki, Finland \\ ${ }^{2}$ Department of Civil and Industrial Engineering, University of Pisa, Pisa, Italy \\ Correspondence to: P. Janhunen (pekka.janhunen@ fmi.fi)
}

Received: 24 May 2012 - Published in Geosci. Instrum. Method. Data Syst. Discuss.: 10 July 2012

Revised: 12 November 2012 - Accepted: 28 January 2013 - Published: 19 February 2013

\begin{abstract}
The electric solar wind sail (E-sail) is a new type of propellantless propulsion system for Solar System transportation, which uses the natural solar wind to produce spacecraft propulsion. The E-sail consists of thin centrifugally stretched tethers that are kept charged by an onboard electron gun and, as such, experience Coulomb drag through the high-speed solar wind plasma stream. This paper discusses a mass breakdown and a performance model for an E-sail spacecraft that hosts a mission-specific payload of prescribed mass. In particular, the model is able to estimate the total spacecraft mass and its propulsive acceleration as a function of various design parameters such as the number of tethers and their length. A number of subsystem masses are calculated assuming existing or near-term E-sail technology. In light of the obtained performance estimates, an Esail represents a promising propulsion system for a variety of transportation needs in the Solar System.
\end{abstract}

\section{Introduction}

The electric solar wind sail (E-sail) is an innovative deep space propulsion concept that uses the solar wind dynamic pressure for generating thrust without the need of reaction mass (Janhunen, 2006, 2009; Janhunen et al., 2010). The Esail spacecraft is spun around its symmetry axis and uses the centrifugal force to deploy and stretch out a number of thin, long and conducting tethers, which are kept in a high positive potential by an onboard electron gun pumping out the negative charge from the system (Janhunen et al., 2010). The latter compensates the electron current gathered by the conducting tethers from the surrounding solar wind plasma. The charged tethers experience Coulomb drag with the highspeed solar wind plasma stream and, thus, generate a propul- sive thrust that is mechanically transmitted to the spacecraft by a slight bending of the tethers perpendicular to their spin plane (Fig. 1).

Our reference full-scale E-sail propulsion system comprises $2000 \mathrm{~km}$ of total main tether length (for example 100 tethers, each one being $20 \mathrm{~km}$ long), with $25 \mathrm{kV}$ tether voltage, $960 \mathrm{~W}$ electron gun power consumption and $1.16 \mathrm{~N}$ nominal thrust at $1 \mathrm{AU}$ from the Sun (Janhunen et al., 2010). If the main tethers are sufficiently long such that the electric potential structure overlapping between them is negligible, the propulsive thrust varies as $1 / r$, where $r$ is the Sun-spacecraft distance (Janhunen, 2009). Note, for comparison, that in the classical photonic solar sail (Wright, 1992; McInnes, 1999) the propulsive thrust decreases more rapidly (that is, as $1 / r^{2}$ ) with the solar distance. Therefore the Esail concept is especially attractive for a mission towards the outer Solar System, such as a Jupiter rendezvous (Quarta et al., 2011) or a mission towards the heliopause (Quarta and Mengali, 2010) and the Solar System boundaries.

The previous assertion about the overlapping negligibility between electric potential structures of different tethers can be justified as follows. At $1 \mathrm{AU}$ the potential structure radius is $\sim 100 \mathrm{~m}$ under average solar wind conditions (Janhunen, 2009). In all E-sail models considered in this paper the distance between the tether tips is $2 \pi \times 20 \mathrm{~km} / 100=$ $1257 \mathrm{~m}$. Thus, under usual conditions, overlapping affects about $200 \mathrm{~m} / 1257 \mathrm{~m}=16 \%$ of the tether length. The electric potential structures scale as proportional to the solar distance $r$ because they are proportional to the plasma Debye length which goes as $\sim 1 / \sqrt{n}$, where $n$ is the plasma density and $n \sim 1 / r^{2}$. Therefore, at 4 AU the overlapping can affect $\sim 64 \%$ of the tether length. Near the main spacecraft, where the tethers are close one to the other, they form an effectively impenetrable obstacle to solar wind ions such that ions are 


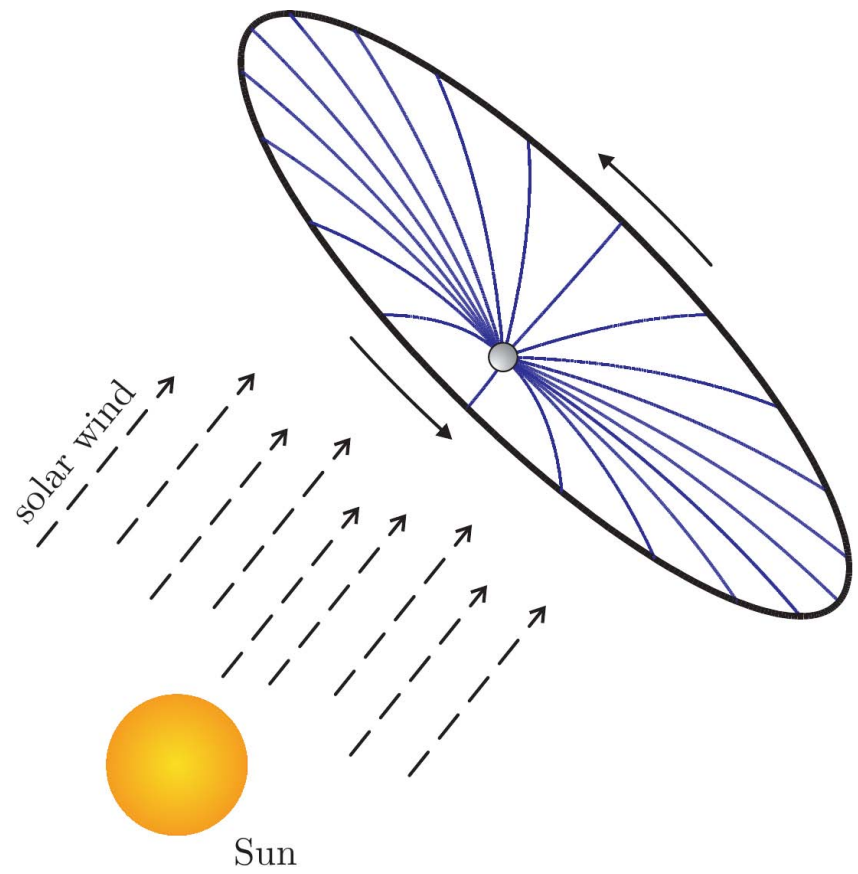

Fig. 1. Schematic view of a spinning E-sail.

reflected back. A model of how the thrust behaves inside the overlap region is not yet available. We roughly estimate that on average, within the overlapping region, the thrust is $60 \%$ of the free tether value. This implies that at $1 \mathrm{AU}$ the thrust would be reduced by $\sim 6 \%$ by the overlapping effect and at 4 AU the reduction would be $\sim 25 \%$.

The E-sail propulsive thrust per unit length (of a main tether) is about $580 \mathrm{nN} \mathrm{m}^{-1}$ so that, for example, a $20 \mathrm{~km}$ long tether gathers about $11.6 \mathrm{mN}$ of thrust from the surrounding solar wind plasma (Janhunen et al., 2010). The previous thrust estimate at $1 \mathrm{AU}$ corresponds to an average solar wind. Actually, the solar wind properties vary widely along, basically, all relevant timescales. However, due to certain plasma physical effects such as Debye length scaling, the E-sail propulsive thrust tends to vary much less than the solar wind dynamic pressure when a simple constant power strategy is applied to adjust the tether voltage in response to solar wind density variations (Toivanen and Janhunen, 2009).

The spacecraft, with its attached main tethers, is spun so that the centrifugal force overcomes the propulsive thrust by a factor of about 5. Accordingly, each main tether has to withstand about $5 \mathrm{cN}(\mathrm{cN}=$ centinewton = about 1 gram's weight in Earth's gravity) continuous pull force without breaking. In addition, the main tethers must survive the micrometeoroid impacts over the mission's lifetime (Hoyt and Forward, 2000), whose maximum reference value is about ten years. These requirements are filled with sufficient margin by a four-line Heytether (Seppänen et al., 2011), produced by ultrasonic bonding from $25 \mu \mathrm{m}$ and $50 \mu \mathrm{m}$ aluminium wires (Kurppa et al., 2010). A Heytether consists of one parallel wire to which several (by default 3) loop wires are bonded to the base wire at regular, mutually interleaving intervals. In terms of micrometeoroid tolerance, the four-wire Heytether is roughly equivalent to the criss-crossed four-wire Hoytether (Hoyt and Forward, 2000), but is easier to manufacture by our methods because only one base wire is needed.

Assuming ten years of flight time with full thrust of $1 \mathrm{~N}$ at about $1 \mathrm{AU}$, an E-sail propulsion system produces a total impulse of about $300 \mathrm{MNs}$. This value is equivalent to the total impulse produced by a high-thrust propulsion system, for example, a chemical rocket with a specific impulse of $300 \mathrm{~s}$ burning 100 tonnes (t) of propellant, or an electric thruster with a specific impulse of $3000 \mathrm{~s}$ that uses $10 \mathrm{t}$ of propellant.

The propulsive acceleration and the corresponding mission performance in terms of flight time depend on both the payload mass and the E-sail design parameters. In order to evaluate the actual E-sail capabilities in a deep-space next generation mission, it is therefore important to have a parametric model that is able to model the propulsion system performance as a function of its (main) design parameters. The purpose of this paper is to develop such a parametric model. The new mathematical model deepens and updates the previous simplified approach of Mengali et al. (2008).

The fact that the E-sail spins slowly has some implications for the payload, especially to imaging science instruments requiring a combination of accurate pointing and lengthy exposure. Specific technical solutions such as despun platforms are available to mitigate or eliminate these potential issues. Analyzing such matters is outside the scope of this paper.

\section{Scalable E-sail mathematical model}

We now consider a parametric model for mass budgeting of E-sail missions of different sizes, see e.g. Larson and Wertz (1999) for the general approach. Consider an E-sail propulsion system, consisting of a main body and $N$ main tethers, each one with length $L$. A remote unit (RU) is placed at the tip of each tether, see Fig. 2. Every RU comprises two reels for deploying an auxiliary tether, as discussed next, and a thruster unit for controlling the main tether's angular velocity. The main spacecraft and the tether rig spin slowly to keep the tethers taut, a typical spin period being some tens of minutes. The reason for including the auxiliary tethers is that they keep the tether rig dynamically stable without the need of active control (Janhunen et al., 2010). The motivation for including RUs is to host the auxiliary tether reels and small thruster, whose purpose is to generate the initial angular momentum and possibly to control the spin rate later during flight, if needed.

The total spacecraft mass can be thought of as being the sum of the following contributions: (1) mission-specific payload of mass $m_{\text {pay }}$; (2) high voltage subsystem including electron guns; (3) $N$ main tethers of mass $m_{\mathrm{mt}}, N$ main tether reels of mass $m_{\mathrm{mr}}$, and $N$ RUs of mass $m_{\mathrm{ru}}$; (4) auxiliary 


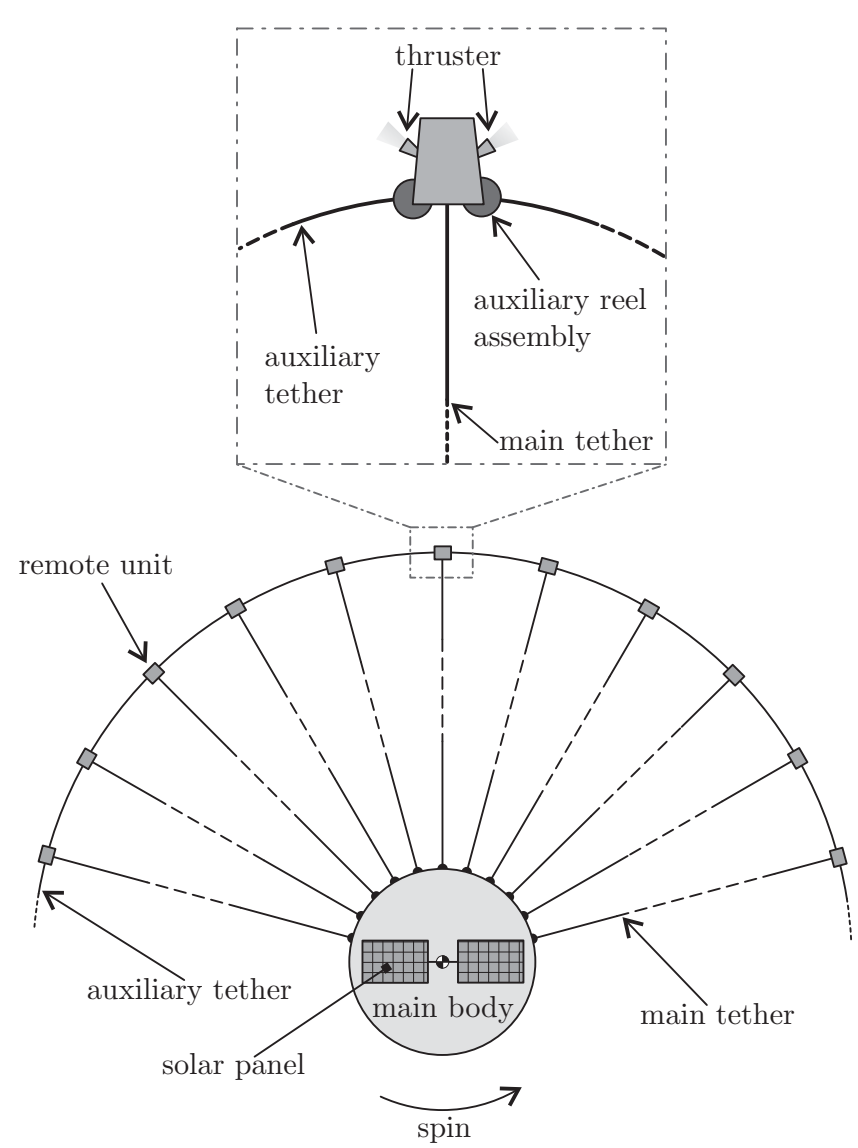

Fig. 2. Schematic view of E-sail with remote unit and auxiliary tethers.

tether of mass $m_{\mathrm{at}}$; (5) tether cameras and E-sail controller; (6) power system with solar panels; (7) telemetry system with antennas; (8) thermal control subsystem; (9) attitude control system (ACS); and (10) structural mass. The simplified expression for the total spacecraft mass $m$ is thus

$m=\eta_{\mathrm{ma}} \frac{m_{\mathrm{b}}+N\left(m_{\mathrm{mt}}+m_{\mathrm{ru}}\right)+m_{\mathrm{at}}}{\left(1-\eta_{\mathrm{str}}\right)\left(1-\eta_{\mathrm{acs}}\right)}$

where the dimensionless margin coefficient $\eta_{\mathrm{ma}}=1.2$ is introduced to account for a $20 \%$ margin on the actual value, while $\eta_{\text {str }}$ and $\eta_{\text {acs }}$ model the structural mass fraction and the ACS mass fraction of the spacecraft's total mass.

Each term in the numerator of the right hand side of Eq. (1) can be expressed as a function of the system's parameters, as will be discussed below. The term $m_{\mathrm{b}}$ indicates the mass of the functional components of the main body of the spacecraft, given by

$$
\begin{aligned}
& m_{\mathrm{b}}= \\
& \frac{m_{\mathrm{gc}}+m_{\mathrm{vs}}+n_{\mathrm{ca}} m_{\mathrm{ca}}+n_{\mathrm{eg}} m_{\mathrm{eg}}+m_{\mathrm{sa}}+\frac{m_{\mathrm{pay}}}{1-\eta_{\mathrm{tms}}}+N m_{\mathrm{mr}}}{1-\eta_{\mathrm{tcs}}} .
\end{aligned}
$$

The mass of the high voltage source $\left(m_{\mathrm{vs}}\right)$, guidance computer $\left(m_{\mathrm{gc}}\right)$, tether cameras $\left(n_{\mathrm{ca}} \cdot m_{\mathrm{ca}}\right)$, electron guns $\left(n_{e g}\right.$. $\left.m_{\text {eg }}\right)$, solar array power system $\left(m_{\mathrm{sa}}\right)$, the telemetry system equipped payload $\left(m_{\text {pay }} /\left(1-\eta_{\mathrm{tms}}\right)\right)$ and the main tether reels $\left(N \cdot m_{\mathrm{mr}}\right)$ are discussed in the following subsections, along with the other mass terms appearing in Eq. (1).

Note that the term "payload" in this paper refers to the payload instruments and the telemetry system, but it does not include the power system, structure or thermal control subsystems. The rationale for lumping the telemetry system with the payload is that the payload drives telemetry requirements, not the E-sail. On the other hand, the power system is kept separate because, typically, the payload uses only little power during the cruise phase and thus it makes sense to share the power system hardware between the E-sail and the payload.

\subsection{High voltage subsystem}

We assume $n_{\mathrm{eg}}=3$ redundant electron guns, each one providing a beam power $P_{\mathrm{eg}}$ and having mass $m_{\mathrm{eg}}=\gamma_{\mathrm{eg}} P_{\mathrm{eg}}$, where the gun specific mass is $\gamma_{\mathrm{eg}}=1.0 \mathrm{~kg} \mathrm{~kW}^{-1}$ (Zavyalov et al., 2006). We assume $100 \%$ gun efficiency and neglect the low voltage cathode heating power. The electric power $P_{\text {eg }}$ varies with the distance $r$ from the Sun and can be related to the total length $N L$ of the main tethers through a linear power density $\beta$, whose value essentially depends (Mengali et al., 2008) on the main tethers voltage and on the Heytether (Seppänen et al., 2011) total surface area. In particular, using the current Heytether configuration, the expression for the linear power density is

$\beta=2 n_{\oplus} \sqrt{\frac{2 e^{3} V_{0}^{3}}{m_{e}}}\left[R_{1}+(3 \pi / 2) R_{2}\right]$,

where $V_{0}$ is the nominal voltage of the main tethers, $n_{\oplus}=$ $7.6 \times 10^{6} \mathrm{~m}^{-3}$ is the nominal solar wind density at $r=r_{\oplus} \equiv$ $1 \mathrm{AU}, e$ is the electron charge, and $m_{e}$ is the electron mass. For example, assuming $V_{0}=25 \mathrm{kV}$ and the previous tether dimensions $\left(R_{1}=25 \mu \mathrm{m}\right.$ and $\left.R_{2}=12.5 \mu \mathrm{m}\right)$, Eq. (3) provides a linear power density $\beta \simeq 0.4790 \mathrm{~W} \mathrm{~km}^{-1}$.

Taking into account a reference condition that corresponds to the minimum Sun-spacecraft distance $r_{\min }=0.9 \mathrm{AU}$, a conservative estimate of the electric power required by the electron gun is

$P_{\text {eg }}=N L \beta\left(r_{\oplus} / r_{\min }\right)^{2}$.

Even though the solar wind density $n$ exhibits large natural variations, a simple strategy of varying the tether voltage $V$ away from the nominal $V_{0}$, such that $P_{\text {eg }}$ is constant, is quite effective for maintaining constant the daily, weekly or monthly averaged thrust at a given solar distance $r$ (Toivanen and Janhunen, 2009).

Two plasma physical effects are responsible for this, at first, surprising behavior. The first one is that the thrust is proportional to the total tether length times the tether's electron 


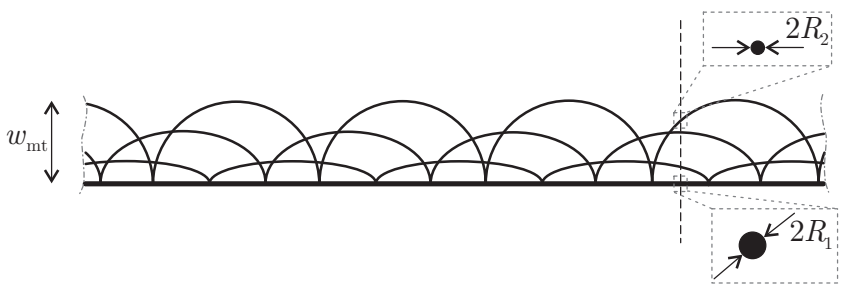

Fig. 3. Four-wire Heytether scheme.

sheath width. For a fixed voltage, the latter is proportional to the solar wind plasma Debye length, which, in turn, is proportional to $1 / \sqrt{n}$. As a result (Janhunen, 2009), the thrust is approximately linearly proportional to the tether voltage $V$, but it has only a square root dependence on the solar wind dynamic pressure $P_{\mathrm{dyn}}=m_{p} n v^{2}$, where $v$ is the solar wind speed and $m_{p}$ is the proton mass. The second effect is that, because the tether current is proportional to $n \sqrt{V}, V$ must be varied as $n^{-2 / 3}$ in order to maintain $P_{\text {eg }}$ constant. When combined, these two effects imply that under a constant $P_{\text {eg }}$ strategy the thrust is proportional to $n^{1 / 6} v$, i.e. the thrust depends only weakly on the solar wind density. Furthermore, solar wind variations of $n$ and $v$ are typically anticorrelated, and this tends to further reduce the thrust fluctuations.

There are several methods on how high voltage distribution (and grounding plan) can be obtained. One way is to have a relatively low energy (e.g. $1 \mathrm{kV})$ electron gun connected to a common internal bus that maintains the electron gun at its voltage. Each tether can then have its own small, high voltage source, thus allowing an arbitrary differential modulation of tether voltages and no need for high voltage switches, resistors, potentiometers or cables. The high voltage source mass is assumed to be $m_{\mathrm{vs}}=\gamma_{\mathrm{vs}} P_{\mathrm{eg}}$, where $\gamma_{\mathrm{vs}}=20 \mathrm{~kg} \mathrm{~kW}^{-1}$ is the specific mass of the high voltage generator. For example, Ultravolt (http://www.ultravolt.com) makes $30 \mathrm{~W} \mathrm{kV}^{-1}$ vacuum compatible DC voltage source model 35A24-P30 with a $\gamma_{\mathrm{vs}}$ of $14.2 \mathrm{~kg} \mathrm{~kW}^{-1}$ and an efficiency of $70 \%$. Therefore a value of $20 \mathrm{~kg} \mathrm{~kW}^{-1}$ seems to be a reasonable value, even though it may require some customization effort. As for the space qualified high voltage systems, electron guns with up to $40 \mathrm{kV}$ accelerating voltage have been successfully operated on sounding rockets for scientific purposes (Nemzek and Winckler, 1991).

\subsection{Main tethers}

The main tether is a four-wire aluminium (density $\rho_{\mathrm{Al}}=$ $2700 \mathrm{~kg} \mathrm{~m}^{-3}$ ) Heytether (Seppänen et al., 2011), composed of a (straight) base wire of radius $R_{1}=25 \mu \mathrm{m}$, and three (approximately semicircular) loop wires of radius $R_{2}=$ $12.5 \mu \mathrm{m}$, the latter being ultrasonically bonded to the former (Kurppa et al., 2010), see Fig. 3.

The mass per unit length of the main tether is $\lambda_{\mathrm{mt}}=$ $\rho_{\mathrm{Al}} \pi\left[R_{1}^{2}+(3 \pi / 2) R_{2}^{2}\right] \simeq 1.155 \times 10^{-5} \mathrm{~kg} \mathrm{~m}^{-1}$. Therefore, the tether mass $m_{\mathrm{mt}}$ depends linearly on $L$ according to the simple relationship

$m_{\mathrm{mt}}=\lambda_{\mathrm{mt}} L$.

\subsection{Main tether reel assembly}

The main tether reel assembly is a motorized mechanism that holds the reeled tether inside, and deploys it in orbit. Its mass is estimated to be

$m_{\mathrm{mr}}=m_{\mathrm{mr} 0}+\rho_{\mathrm{mr}} \frac{V_{\mathrm{mt}}}{\eta_{\mathrm{mr}}}$,

where $m_{\mathrm{mr} 0}=0.1 \mathrm{~kg}$ corresponds to the mass of the motorized reel assembly in case of a short tether such as that used in ESTCube-1 (Janhunen et al., 2010; Pajusalu et al., 2012) and Aalto-1 (Praks et al., 2011; Näsilä et al., 2011) CubeSat missions, $\rho_{\mathrm{mr}}=500 \mathrm{~kg} \mathrm{~m}^{-3}$ is the assumed mass density of the reel structure with respect to its contained volume, $V_{\mathrm{mt}}=m_{\mathrm{mt}} / \rho_{\mathrm{Al}}$ is the solid aluminium volume of the main tether and $\eta_{\mathrm{mr}}=0.3$ is the packaging factor of the reeled tether.

\subsection{Auxiliary tether}

The auxiliary tether is manufactured using Kapton (with a density $\rho_{\mathrm{Ka}}=1420 \mathrm{~kg} \mathrm{~m}^{-3}$ ) and is used to connect the RUs for avoiding collisions between adjacent tethers (Janhunen et al., 2010). Assuming that the auxiliary tether is constituted by a rectangular section of height $h_{\mathrm{at}}=12.7 \mu \mathrm{m}$ and width $w_{\text {at }}=3 \mathrm{~cm}$, its linear density is $\lambda_{\text {at }}=\eta_{p} \rho_{\mathrm{Ka}} h_{\text {at }} w_{\text {at }}=$ $2.705 \times 10^{-4} \mathrm{~kg} \mathrm{~m}^{-1}$ where $\eta_{\mathrm{p}}=0.5$ is a dimensionless coefficient that models the perforation of the auxiliary tether's stripe required to produce a proper amount of elasticity. The length of the auxiliary tether is approximately equal to the length of a circumference of radius $L$. The total auxiliary tether mass is thus

$m_{\mathrm{at}}=\lambda_{\mathrm{at}} 2 \pi L$.

\subsection{Remote units}

Each RU hosts a thruster for initiating and (possibly later) controlling the tether rig's spin. It also includes the reels from which the auxiliary tethers are deployed. Two thruster options are being considered in more detail, a cold gas thruster of Nanospace and an ionic liquid FEEP (Field Emission Electric Propulsion) thruster of Alta (Marcuccio et al., 2011). The cold gas thruster can produce a total impulse sufficient for the required initial spin and for the small spin rate adjustments during flight operations. The FEEP thruster, on the other hand, has a total impulse capability sufficient for controlling the spin to counteract the Coriolis acceleration that results from orbiting around the Sun with an inclined sail (Toivanen and Janhunen, 2012).

Prototypes of cold gas thruster and FEEP thruster equipped RUs have been designed at Ångström Space Technology Centre (Wagner et al., 2012). The wet mass of a 
FEEP-unit is $0.880 \mathrm{~kg}$, whose auxiliary tether reel system's share is $0.135 \mathrm{~kg}$. The mass of the ionic liquid propellant is $0.07 \mathrm{~kg}$ and the total impulse capability $2000 \mathrm{Ns}$. The wet mass of a cold-gas unit is $0.613 \mathrm{~kg}$, of which $0.05 \mathrm{~kg}$ is propellant, and the total impulse capability is $40 \mathrm{Ns}$.

For conservative purposes, here we assume that each RU contains a FEEP thruster that is mounted either along the spin accelerating direction or along the decelerating direction, see Fig. 2. Therefore there are two subtypes of RUs, which are otherwise identical except being mirror images of each other in the left-right direction. In a baseline configuration, accelerating and braking thrusters are alternated on adjacent RUs. More general arrangements could also be considered in specific missions. Accordingly, the RU's mass with a FEEP unit is parameterized as

$m_{\mathrm{ru}}=m_{\mathrm{ru} 0}+\frac{m_{\mathrm{at}}}{\rho_{\mathrm{Ka}}} \rho_{\mathrm{ar}}$,

where $m_{\mathrm{ru} 0}=0.745 \mathrm{~kg}$ and $\rho_{\mathrm{ar}}=282 \mathrm{~kg} \mathrm{~m}^{-3}$.

\subsection{Tether cameras and controller}

To find out the actual position of each RU at the main tether tips, a number $n_{\mathrm{ca}}=12$ of cameras along the perimeter of the main spacecraft are used. Each camera has a mass of $m_{\mathrm{ca}}=$ $0.04 \mathrm{~kg}$ (Pappa et al, 2004). Each RU has an optical beacon transmitting a unique optical coding so that the unit can be identified by the cameras. The E-sail also needs a guidance computer to which a mass $m_{\mathrm{gc}}=1 \mathrm{~kg}$ is allocated, including the radiation shielding. Since the tether rig moves slowly, a moderate amount of computing power is sufficient.

\subsection{Power generation subsystem}

The power generation subsystem includes solar panels with their deployment mechanism as well as a power processing unit that produces bus voltage and, very likely, a battery pack.

For a baseline deep space mission, where the Sunspacecraft distance ranges between about $0.9 \mathrm{AU}$ and $4 \mathrm{AU}$, rather large solar panels are typically needed to provide the payload with a sufficient power up to the aphelion radius. It is assumed that during the cruise phase both payload and telemetry instruments are in idle (or keep-alive) mode, with a specific power consumption of $0.1 \mathrm{~W} \mathrm{~kg}^{-1}$, while during the operating mode (that is, when the E-sail is turned off) the power consumption is $1 \mathrm{~W} \mathrm{~kg}^{-1}$. To be conservative, we assume that the E-sail could also use $10 \mathrm{~W}$ of base power in addition to the electron gun requirement, even when it is turned off. Note that the electric power required by the electron gun varies with the solar distance as $1 / r^{2}$, that is, in the same way as the illumination of the solar panels.

For sizing the payload, it turns out that the power system requirements are always driven by the payload requirements at $4 \mathrm{AU}$ (aphelion distance), and not by the E-sail requirements during the cruise phase. Hence, the power sys- tem has to provide $1 \mathrm{~W} \mathrm{~kg}^{-1}$ for the payload and telemetry units at $4 \mathrm{AU}$, plus $10 \mathrm{~W}$ of base power for the E-sail. Note that at closer solar distances the power system produces more power than is actually necessary. We assume a specific mass value of $\gamma_{\mathrm{sa}}=10 \mathrm{~kg} \mathrm{~kW}^{-1}$ for the power subsystem as a whole, when the reference $\mathrm{kW}$-value is the full power gathered at $0.9 \mathrm{AU}$. This is motivated by the fact that the full panel power at $0.9 \mathrm{AU}$ does not need to be processed by the power processing unit. It has only to provide enough power to the payloads and to guarantee the solar panels health. We also assume an end-of-life solar panel degradation factor of $\eta_{\mathrm{sa}}=1.2$ : where power produced by the panels at end-of-life is assumed to be $\eta_{\mathrm{sa}}$ times less than at beginning of life.

Accordingly, the power produced by the solar arrays at the maximum distance $r_{\max }=4 \mathrm{AU}$ is

$P_{\mathrm{sa}}^{\mathrm{rmax}}=\eta_{\mathrm{sa}}\left[P_{\mathrm{o}}+\max \left(\eta_{\mathrm{vs}} P_{\mathrm{eg}}\left(\frac{r_{\text {min }}}{r_{\mathrm{max}}}\right)^{2}+\eta_{\mathrm{ka}} P_{\text {pay }}, P_{\text {pay }}\right)\right]$,

where $P_{\text {pay }}=m_{\text {pay }} / \gamma_{\text {pay }}$ is the payload required power, $\eta_{\mathrm{ka}}=$ 0.1 is the idle versus duty power ratio of both the payload and the telemetry systems, and $\eta_{\mathrm{vs}}=1.25$ is the assumed overhead factor (reciprocal of the efficiency) of the HV source. Correspondingly, the needed solar array power at the minimum distance $r_{\min }=0.9 \mathrm{AU}$ is

$P_{\mathrm{sa}}^{\mathrm{rmin}}=\eta_{\mathrm{sa}}\left[P_{\mathrm{o}}+\max \left(\eta_{\mathrm{vs}} P_{\mathrm{eg}}+\eta_{\mathrm{ka}} P_{\mathrm{pay}}, P_{\mathrm{pay}}\right)\right]$.

From the $1 / r^{2}$ scaling of the solar radiation flux, it follows that the needed maximum capacity of the solar arrays scaled to $r_{\min }$ is

$P_{\mathrm{sa}}=\max \left[P_{\mathrm{sa}}^{\mathrm{rmin}}, P_{\mathrm{sa}}^{\mathrm{rmax}}\left(r_{\max } / r_{\min }\right)^{2}\right]$.

Finally, the mass of the power subsystem is given by $m_{\mathrm{sa}}=$ $\gamma_{\mathrm{sa}} P_{\mathrm{sa}}$. Recall that an underlying assumption in the above formulas is that the science payload is active during coasting phases and dormant during propulsive phases, the dormant payload power being factor $\eta_{\mathrm{ka}}$ times the active payload power.

\subsection{Telemetry, ACS, thermal and structure}

We assume that the telemetry subsystem mass is related to the payload such that the telemetry subsystem plus payload mass is given by $m_{\text {pay }} /\left(1-\eta_{\text {tms }}\right)$, where the telemetry mass fraction is $\eta_{\mathrm{tms}}=0.2$. This choice is qualitatively motivated by the fact that each scientific instrument included in the payload usually generates data that must be transmitted by the telemetry system. If a payload needs more telemetry capability than is assumed here, one has to reserve extra mass for it from the payload budget. Our results concerning the Esail mass fraction are not sensitive to the previous parametric choice.

The E-sail requires a service from the satellite's attitude control system (ACS) for pointing the spin axis towards the 
Table 1. Physical reference data.

\begin{tabular}{|c|c|c|}
\hline Parameter & Symbol & Value \\
\hline Thrust/length/voltage & $f_{0}$ & $24.2 \mathrm{nN} /(\mathrm{kV} \mathrm{m})$ \\
\hline Nominal tether voltage & $V_{0}$ & $25 \mathrm{kV}$ \\
\hline Thrust per length & $f_{V}$ & $24.2 \mathrm{nN} \mathrm{m}^{-1}$ \\
\hline Aux. tether thickness & $h_{\text {at }}$ & $12.7 \mu \mathrm{m}$ \\
\hline Main reel base mass & $m_{\mathrm{mr} 0}$ & $0.1 \mathrm{~kg}$ \\
\hline Tether camera mass & $m_{\mathrm{ca}}$ & $0.04 \mathrm{~kg}$ \\
\hline Number of tether cameras & $n_{\mathrm{ca}}$ & 12 \\
\hline E-sail computer mass & $m_{\mathrm{gc}}$ & $1 \mathrm{~kg}$ \\
\hline FEEP Remote Unit base $\mathrm{m}$. & $m_{\mathrm{ru} 0}$ & $0.745 \mathrm{~kg}$ \\
\hline Number of electron guns & $n_{\text {eg }}$ & 3 \\
\hline 1 AU solar wind density & $n_{\oplus}$ & $7.6 \mathrm{~cm}^{-3}$ \\
\hline E-sail base power & $P_{\mathrm{O}}$ & $10 \mathrm{~W}$ \\
\hline Heytether base wire radius & $R_{1}$ & $25 \mu \mathrm{m}$ \\
\hline Heytether loop wire radius & $R_{2}$ & $12.5 \mu \mathrm{m}$ \\
\hline Minimum solar distance & $r_{\min }$ & $0.9 \mathrm{AU}$ \\
\hline Maximum solar distance & $r_{\max }$ & $4.0 \mathrm{AU}$ \\
\hline Aux. tether width & $w_{\text {at }}$ & $3 \mathrm{~cm}$ \\
\hline Telemetry mass fraction & $\eta_{\mathrm{tms}}$ & 0.2 \\
\hline ACS mass fraction & $\eta_{\text {acs }}$ & 0.05 \\
\hline Overall mass margin factor & $\eta_{\mathrm{ma}}$ & 1.2 \\
\hline Main tether reel fill factor & $\eta_{\mathrm{mr}}$ & 0.3 \\
\hline Aux. tether perforation & $\eta_{\mathrm{p}}$ & 0.5 \\
\hline Solar panel EOL degrad. fac. & $\eta_{\mathrm{sa}}$ & 1.2 \\
\hline Structural fraction & $\eta_{\mathrm{str}}$ & 0.15 \\
\hline Therm. sys. mass fraction & $\eta_{\mathrm{tcs}}$ & 0.05 \\
\hline HV source loss factor & $\eta_{\mathrm{vs}}$ & 1.25 \\
\hline Payload mass per power & $\gamma_{\text {pay }}$ & $1000 \mathrm{~kg} \mathrm{~kW}^{-1}$ \\
\hline E-gun mass per power & $\gamma_{\mathrm{eg}}$ & $1 \mathrm{~kg} \mathrm{~kW}^{-1}$ \\
\hline Power system mass per power & $\gamma_{\mathrm{sa}}$ & $10 \mathrm{~kg} \mathrm{~kW}^{-1}$ \\
\hline HV source mass per power & $\gamma_{\mathrm{vs}}$ & $20 \mathrm{~kg} \mathrm{~kW}^{-1}$ \\
\hline Aluminium density & $\rho_{\mathrm{Al}}$ & $2700 \mathrm{~kg} \mathrm{~m}^{-3}$ \\
\hline Kapton density & $\rho_{\mathrm{Ka}}$ & $1420 \mathrm{~kg} \mathrm{~m}^{-3}$ \\
\hline Aux. tether reel "density" & $\rho_{\mathrm{ar}}$ & $282 \mathrm{~kg} \mathrm{~m}^{-3}$ \\
\hline Main reel "density" & $\rho_{\mathrm{mr}}$ & $500 \mathrm{~kg} \mathrm{~m}^{-3}$ \\
\hline
\end{tabular}

Sun and starting the spin motion at the beginning of E-sail deployment. Most of the required angular momentum is obtained from RU thrusters, but a small fraction is gotten from the ACS. If the mission calls for accurate manoeuvring near an asteroid or another small body, a micropropulsion system is needed for overcoming a small photonic sail effect of the tethers and for fine orbit control. We assume that the attitude and orbit control system (AOCS) mass is a fraction $\eta_{\text {acs }}=0.05$ of the spacecraft's total mass.

Similarly, the thermal control subsystem mass $m_{\text {tcs }}$ is expressed as a given percentage of the main body mass through the coefficient $\eta_{\mathrm{tcs}}=0.05$. Finally, the structural parts of the main spacecraft including RU launch locks is, by assumption, a fraction $\eta_{\mathrm{str}}=0.15$ of the total mass. The main spacecraft parameters of the mass model are collected in Table 1.
Table 2. Spacecraft mass budget and some other properties for a characteristic acceleration $a_{\oplus}=0.1 \mathrm{~mm} \mathrm{~s}^{-2}$.

\begin{tabular}{lcccc}
\hline Payload $m_{\text {pay }}(\mathrm{kg})$ & 100 & 200 & 500 & 1000 \\
\hline Number of tethers $N$ & 12 & 16 & 24 & 34 \\
Tether length $L(\mathrm{~km})$ & 4.02 & 5.77 & 9.27 & 12.9 \\
E-sail thrust at $1 \mathrm{AU}(\mathrm{N})$ & 0.03 & 0.05 & 0.13 & 0.25 \\
E-sail power $P_{\mathrm{O}}+P_{\mathrm{eg}}(\mathrm{W})$ & 38.5 & 64.6 & 142 & 269 \\
Payload idle power $(\mathrm{W})$ & 12.5 & 25.0 & 62.5 & 125 \\
Payload duty power $(\mathrm{W})$ & 125 & 250 & 625 & 1250 \\
\hline Main tethers $N m_{\mathrm{mt}}(\mathrm{kg})$ & 0.56 & 1.07 & 2.57 & 5.05 \\
Main tether reels $N m_{\mathrm{mr}}(\mathrm{kg})$ & 1.54 & 2.26 & 3.99 & 6.52 \\
Electron guns $3 m_{\mathrm{eg}}(\mathrm{kg})$ & 0.09 & 0.16 & 0.39 & 0.78 \\
HV source $m_{\mathrm{vs}}(\mathrm{kg})$ & 0.57 & 1.09 & 2.63 & 5.17 \\
Cameras and computer $(\mathrm{kg})$ & 1.48 & 1.48 & 1.48 & 1.48 \\
Remote units $N m_{\mathrm{ru}}(\mathrm{kg})$ & 11.7 & 15.8 & 24.1 & 34.0 \\
Auxtethers $(\mathrm{kg})$ & 6.83 & 9.80 & 15.8 & 21.9 \\
Power system $m_{\mathrm{sa}}(\mathrm{kg})$ & 32.0 & 61.6 & 151 & 299 \\
Telemetry system $(\mathrm{kg})$ & 25.0 & 50.0 & 125 & 250 \\
Thermal control $(\mathrm{kg})$ & 8.46 & 16.7 & 41.3 & 82.2 \\
ACS $(\mathrm{kg})$ & 9.90 & 18.9 & 45.7 & 89.8 \\
Structure $(\mathrm{kg})$ & 35.0 & 66.9 & 161 & 317 \\
20\% margin $(\mathrm{kg})$ & 46.6 & 89.2 & 215 & 422 \\
\hline Total without E-sail $(\mathrm{kg})$ & 248 & 490 & 1215 & 2425 \\
E-sail effective $(\mathrm{kg})$ & 31.7 & 45.1 & 74.1 & 110 \\
Total $(\mathrm{kg})$ & 280 & 535 & 1290 & 2535 \\
\hline E-sail mass fraction $(\%)$ & 11.4 & 8.44 & 5.74 & 4.34 \\
E-sail specific acc. $(\mathrm{mm} \mathrm{s}-2)$ & 0.88 & 1.18 & 1.74 & 2.30 \\
\hline
\end{tabular}

\subsection{Characteristic acceleration}

Using Table 1 the total spacecraft mass given by Eq. (1) can be computed in terms of four design parameters, namely $N, L, V_{0}, m_{\text {pay }}$.

The spacecraft characteristic acceleration, that is, the maximum propulsive acceleration at a reference distance $r_{\oplus}$ from the Sun, can be similarly expressed as a function of the same four parameters. In fact, the thrust per unit main-tether-length at a distance of 1 AU from the Sun is (Janhunen et al., 2010)

$f=f_{V} V_{0}-f_{0}$,

where $f_{0} \equiv 24.16 \mathrm{nNm}^{-1}$ and $f_{V} \equiv 24.16 \mathrm{nNm}^{-1} \mathrm{kV}^{-1}$. The spacecraft characteristic acceleration is therefore

$a_{\oplus}=\frac{f N L}{m}$.

\section{Results}

Tables 2, 3 and 4 show the spacecraft mass budget and some other fundamental parameters corresponding to a characteristic acceleration $a_{\oplus} 0.1,0.3$ and $1 \mathrm{~mm} \mathrm{~s}^{-2}$. In each 
Table 3. Same as Table 2, but for a characteristic acceleration $a_{\oplus}=$ $0.3 \mathrm{~mm} \mathrm{~s}^{-2}$.

\begin{tabular}{lcccc}
\hline Payload $m_{\text {pay }}(\mathrm{kg})$ & 100 & 200 & 500 & 1000 \\
\hline Number of tethers $N$ & 16 & 24 & 36 & 50 \\
Tether length $L(\mathrm{~km})$ & 6.38 & 8.02 & 12.7 & 17.9 \\
E-sail thrust at $1 \mathrm{AU}(\mathrm{N})$ & 0.06 & 0.11 & 0.27 & 0.52 \\
E-sail power $P_{\mathrm{O}}+P_{\text {eg }}(\mathrm{W})$ & 70.3 & 124 & 281 & 540 \\
Payload idle power $(\mathrm{W})$ & 12.5 & 25.0 & 62.5 & 125 \\
Payload duty power $(\mathrm{W})$ & 125 & 250 & 625 & 1250 \\
\hline Main tethers $N m_{\mathrm{mt}}(\mathrm{kg})$ & 1.18 & 2.22 & 5.29 & 10.3 \\
Main tether reels $N m_{\mathrm{mr}}(\mathrm{kg})$ & 2.33 & 3.77 & 6.87 & 11.4 \\
Electron guns $3 m_{\mathrm{eg}}(\mathrm{kg})$ & 0.18 & 0.34 & 0.81 & 1.59 \\
HV source $m_{\mathrm{vs}}(\mathrm{kg})$ & 1.21 & 2.28 & 5.42 & 10.6 \\
Cameras and computer $(\mathrm{kg})$ & 1.48 & 1.48 & 1.48 & 1.48 \\
Remote units $N m_{\mathrm{ru}}(\mathrm{kg})$ & 16.2 & 23.3 & 35.4 & 49.3 \\
Auxtethers $(\mathrm{kg})$ & 10.8 & 13.6 & 21.6 & 30.4 \\
Power system $m_{\mathrm{sa}}(\mathrm{kg})$ & 32.0 & 61.6 & 151 & 299 \\
Telemetry system $(\mathrm{kg})$ & 25.0 & 50.0 & 125 & 250 \\
Thermal control $(\mathrm{kg})$ & 8.54 & 16.8 & 41.6 & 82.8 \\
ACS $(\mathrm{kg})$ & 10.5 & 19.8 & 47.1 & 91.9 \\
Structure $(\mathrm{kg})$ & 37.0 & 69.7 & 166 & 324 \\
20\% margin $(\mathrm{kg})$ & 49.3 & 93.0 & 221 & 433 \\
\hline Total without E-sail $(\mathrm{kg})$ & 248 & 490 & 1215 & 2425 \\
E-sail effective $(\mathrm{kg})$ & 47.8 & 68.2 & 113 & 171 \\
Total $(\mathrm{kg})$ & 296 & 558 & 1329 & 2596 \\
\hline E-sail mass fraction $(\%)$ & 16.2 & 12.2 & 8.52 & 6.58 \\
E-sail specific acc. $(\mathrm{mm} \mathrm{s}-2)$ & 1.24 & 1.64 & 2.35 & 3.04 \\
\hline
\end{tabular}

case (label "Total" in the tables) the number of tethers, an even integer, was optimized for minimizing the total spacecraft mass, and the tether length was iteratively adjusted for each $N$ until the desired characteristic acceleration was obtained. Note that the tether length was restricted to a maximum value of $20 \mathrm{~km}$ and the number of tethers to 100 . The "Total without E-sail" values were obtained by using the same mass formula, but enforcing the conditions $N=0$ and $L=0$. This represents a spacecraft with the same payload mass and other functionalities, but without on-orbit propulsive capabilities. The E-sail mass fraction is the effective mass divided by the total spacecraft mass, and the E-sail specific acceleration is the propulsive thrust at $1 \mathrm{AU}$ divided by its effective mass. Tables 2, 3 and 4 show some characteristic trends that can be summarized as follows.

1. When the E-sail size increases, its specific acceleration improves and the E-sail mass fraction decreases. This is because the main tether reels and RUs have, by assumption, a certain base mass even in the limit of short main and auxiliary tethers. By redesigning and miniaturizing these items, the E-sail specific acceleration could probably be improved for small sizes. On the other hand, the trend would probably not continue to even larger sizes, because for tethers longer than $20-30 \mathrm{~km}$, their
Table 4. Same as Table 2, but for a characteristic acceleration $a_{\oplus}=$ $1 \mathrm{~mm} \mathrm{~s}^{-2}$.

\begin{tabular}{lccc}
\hline Payload $m_{\text {pay }}(\mathrm{kg})$ & 100 & 200 & 300 \\
\hline Number of tethers $N$ & 44 & 62 & 86 \\
Tether length $L(\mathrm{~km})$ & 15.3 & 19.4 & 20.0 \\
E-sail thrust at $1 \mathrm{AU}(\mathrm{N})$ & 0.39 & 0.70 & 1.00 \\
E-sail power $P_{\mathrm{O}}+P_{\text {eg }}(\mathrm{W})$ & 409 & 720 & 1026 \\
Payload idle power $(\mathrm{W})$ & 12.5 & 25.0 & 37.5 \\
Payload duty power $(\mathrm{W})$ & 125 & 250 & 375 \\
\hline Main tethers $N m_{\mathrm{mt}}(\mathrm{kg})$ & 7.79 & 13.9 & 19.8 \\
Main tether reels $N m_{\mathrm{mr}}(\mathrm{kg})$ & 9.21 & 14.8 & 20.8 \\
Electron guns $3 m_{\mathrm{eg}}(\mathrm{kg})$ & 1.20 & 2.13 & 3.05 \\
HV source $m_{\mathrm{vs}}(\mathrm{kg})$ & 7.98 & 14.2 & 20.3 \\
Cameras and computer $(\mathrm{kg})$ & 1.48 & 1.48 & 1.48 \\
Remote units $N m_{\mathrm{ru}}(\mathrm{kg})$ & 43.1 & 59.3 & 77.6 \\
Auxtethers $(\mathrm{kg})$ & 26.1 & 32.9 & 34.0 \\
Power system $m_{\mathrm{sa}}(\mathrm{kg})$ & 32.0 & 61.6 & 91.3 \\
Telemetry system $(\mathrm{kg})$ & 25.0 & 50.0 & 75.0 \\
Thermal control $(\mathrm{kg})$ & 9.31 & 18.1 & 26.9 \\
ACS $(\mathrm{kg})$ & 13.8 & 24.7 & 35.3 \\
Structure $(\mathrm{kg})$ & 48.9 & 87.0 & 125 \\
20\% margin $(\mathrm{kg})$ & 65.2 & 116 & 166 \\
\hline Total without E-sail $(\mathrm{kg})$ & 248 & 490 & 732 \\
E-sail effective $(\mathrm{kg})$ & 143 & 206 & 264 \\
Total $(\mathrm{kg})$ & 391 & 696 & 996 \\
\hline E-sail mass fraction $(\%)$ & 36.6 & 29.6 & 26.5 \\
E-sail specific acc. $\mathrm{mm} \mathrm{s}-2)$ & 2.73 & 3.38 & 3.77 \\
\hline
\end{tabular}

tensile strength requirement would start to grow beyond what Heytethers tolerate. If even longer tethers were used, thicker wires or better materials should probably be employed.

2. Tables 2 and 3 show that by moving from $0.1 \mathrm{~mm} \mathrm{~s}^{-2}$ to $0.3 \mathrm{~mm} \mathrm{~s}^{-2}$ of characteristic acceleration, the E-sail's mass fraction increases only slightly. For example, for a $1000 \mathrm{~kg}$ of payload, the spacecraft total mass is about $2535 \mathrm{~kg}$ when $a_{\oplus}=0.1 \mathrm{~mm} \mathrm{~s}^{-2}$, while it is $2596 \mathrm{~kg}$ (only $2.4 \%$ larger) for a three times more capable system $\left(a_{\oplus}=0.3 \mathrm{~mm} \mathrm{~s}^{-2}\right)$. In light of these numbers and assuming the availability of E-sails of different sizes, using the lowest characteristic acceleration $\left(a_{\oplus}=\right.$ $0.1 \mathrm{~mm} \mathrm{~s}^{-2}$ ) might be motivated only if the spacecraft payload is of some bulk material, such as products from asteroid mining, rather than a technical payload.

3. Currently, RUs, auxiliary tethers, main tethers, main tether reels, and the HV subsystem all significantly contribute to the E-sail's effective mass.

For comparative purposes, Table 5 shows the mass breakdown for a spacecraft having the same parameters of Table 4 with the exception that in Table 5 the auxiliary tethers are made of $7.6 \mu \mathrm{m}$ Kapton (instead of $12.7 \mu \mathrm{m}$ ) and that the cold 
Table 5. Same as Table 4, but with thinner auxiliary tethers $(7.6 \mu \mathrm{m})$ and RUs with cold gas thrusters.

\begin{tabular}{lccc}
\hline Payload $m_{\text {pay }}(\mathrm{kg})$ & 100 & 200 & 300 \\
\hline Number of tethers $N$ & 38 & 56 & 80 \\
Tether length $L(\mathrm{~km})$ & 15.6 & 19.5 & 19.9 \\
E-sail thrust at $1 \mathrm{AU}(\mathrm{N})$ & 0.34 & 0.63 & 0.92 \\
E-sail power $P_{\mathrm{O}}+P_{\text {eg }}(\mathrm{W})$ & 360 & 657 & 950 \\
Payload idle power $(\mathrm{W})$ & 12.5 & 25.0 & 37.5 \\
Payload duty power $(\mathrm{W})$ & 125 & 250 & 375 \\
\hline Main tethers $N m_{\mathrm{mt}}(\mathrm{kg})$ & 6.83 & 12.6 & 18.4 \\
Main tether reels $N m_{\mathrm{mr}}(\mathrm{kg})$ & 8.02 & 13.4 & 19.3 \\
Electron guns $3 m_{\text {eg }}(\mathrm{kg})$ & 1.05 & 1.94 & 2.82 \\
HV source $m_{\mathrm{vs}}(\mathrm{kg})$ & 7.00 & 12.9 & 18.8 \\
Cameras and computer $(\mathrm{kg})$ & 1.48 & 1.48 & 1.48 \\
Remote units $N m_{\mathrm{ru}}(\mathrm{kg})$ & 24.5 & 34.7 & 46.3 \\
Auxtethers $(\mathrm{kg})$ & 15.8 & 19.9 & 20.2 \\
Power system $m_{\mathrm{sa}}(\mathrm{kg})$ & 32.0 & 61.6 & 91.3 \\
Telemetry system $(\mathrm{kg})$ & 25.0 & 50.0 & 75.0 \\
Thermal control $(\mathrm{kg})$ & 9.19 & 18.0 & 26.8 \\
ACS $(\mathrm{kg})$ & 12.2 & 22.4 & 32.6 \\
Structure $(\mathrm{kg})$ & 42.9 & 79.2 & 115 \\
20\% margin $(\mathrm{kg})$ & 57.2 & 106 & 154 \\
\hline Total without E-sail $(\mathrm{kg})$ & 248 & 490 & 732 \\
E-sail effective $(\mathrm{kg})$ & 95.2 & 144 & 190 \\
Total $(\mathrm{kg})$ & 343 & 634 & 922 \\
\hline E-sail mass fraction $(\%)$ & 27.7 & 22.7 & 20.6 \\
E-sail specific acc. $(\mathrm{mm} \mathrm{s}-2)$ & 3.60 & 4.40 & 4.85 \\
\hline
\end{tabular}

gas thruster option is taken into account. Recall that the wet mass of the cold gas unit is $0.267 \mathrm{~kg}$ lighter than the FEEP version. The mass of a RU with cold gas thrusters can also be representative of a solar photon blade equipped version of the RU, which has sufficient spin rate control capability for any mission (Janhunen, 2012). Making the auxiliary tethers thinner, favours longer tethers in the mass optimization process, while a reduced RU base mass has the opposite effect. Because the numbers of tethers in Table 5 are smaller than those in Table 4, a reduction of the auxiliary tether thickness has a larger impact than that of changing the RU's thruster class. For the $300 \mathrm{~kg}$ payload case, the net result is a $28 \%$ reduction in the E-sail effective mass and a corresponding increase in the E-sail specific acceleration.

We have thus far assumed that the nominal tether voltage $V_{0}$ (valid for average solar wind conditions) has a fixed value of $25 \mathrm{kV}$. The maximum voltage for which the hardware is designed should be larger, perhaps $40 \mathrm{kV}$, because otherwise the thrust would be decreased when the solar wind density is lower than its average value (Toivanen and Janhunen, 2009). Trading off the hardware voltage limit against other design parameters is outside the scope of this paper, although we will explore the effect of the value of $V_{0}$ at the end of this section.

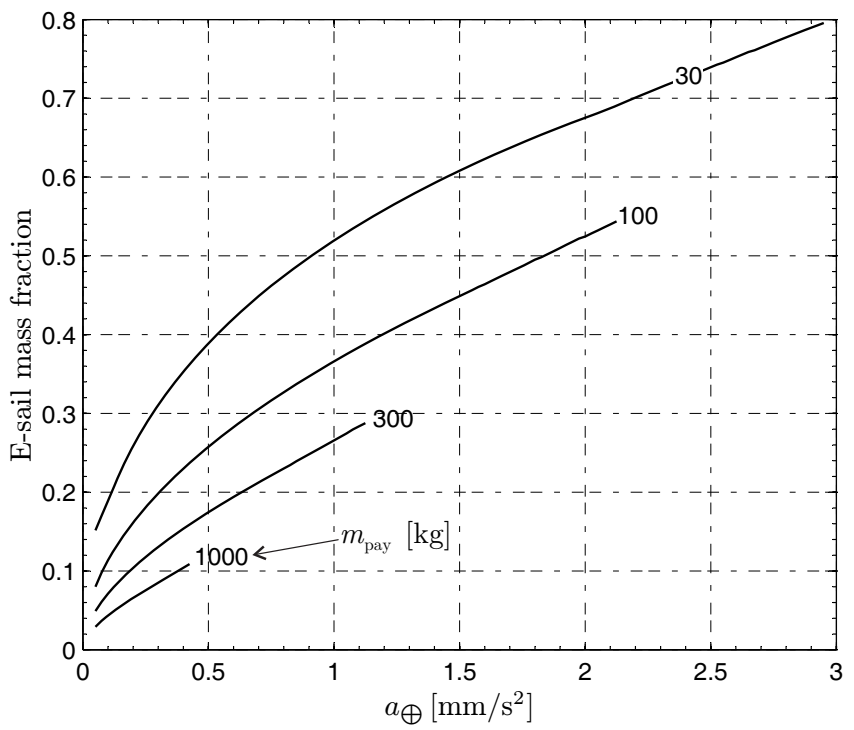

Fig. 4. E-sail mass fraction as a function of $a_{\oplus}$ and $m_{\text {pay }}$.

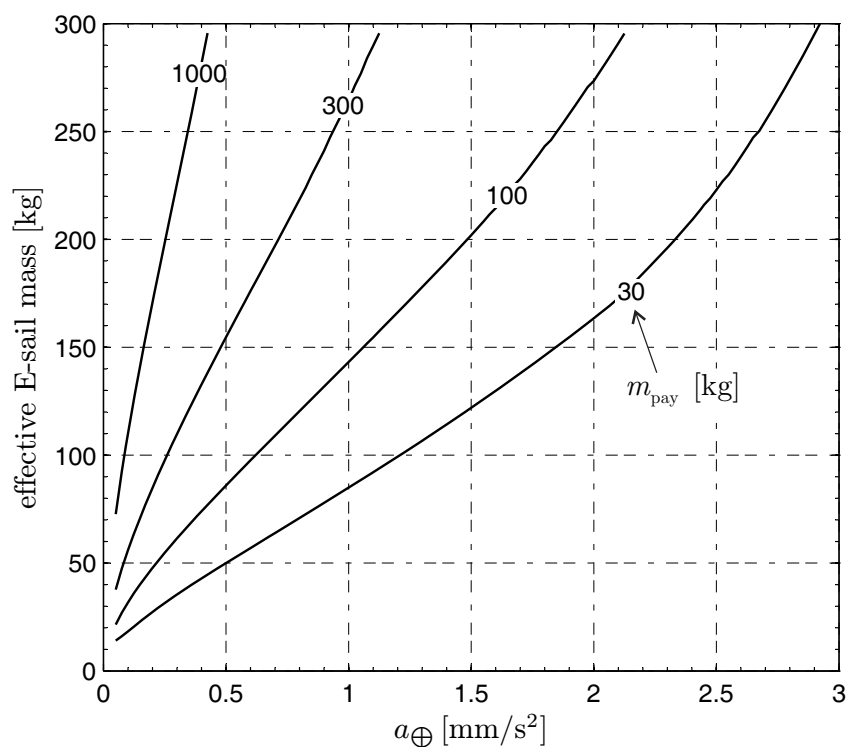

Fig. 5. Effective E-sail mass as a function of $a_{\oplus}$ and $m_{\text {pay }}$.

Figure 4 shows the E-sail mass fraction (effective E-sail mass divided by spacecraft total mass) as a function of the characteristic acceleration, for different payloads $m_{\text {pay }}$ of 30 , 100,300 and $1000 \mathrm{~kg}$. For each payload mass, there exists a maximum characteristic acceleration that can be reached by an E-sail. Recall that, by assumption, the E-sail performance is constrained by a maximum number (100) and length $(20 \mathrm{~km})$ of main tethers.

Figures 5-7 illustrate the corresponding effective E-sail mass, total spacecraft mass and E-sail propulsive thrust, respectively. Figures 4-7 span a wide range of potential applications. Small values of characteristic accelerations with a 


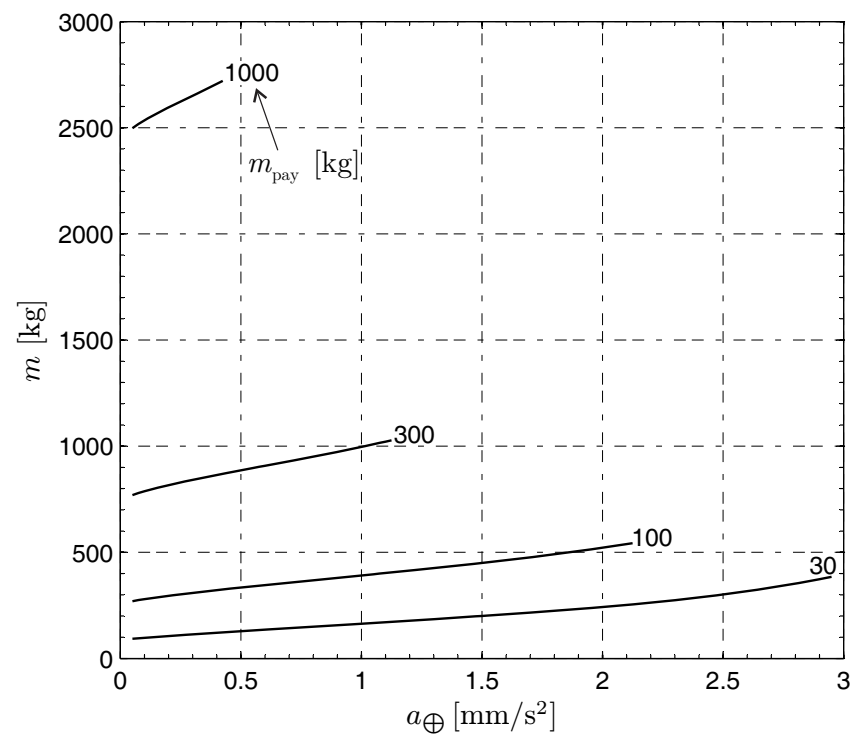

Fig. 6. Spacecraft total mass as a function of $a_{\oplus}$ and $m_{\text {pay }}$.

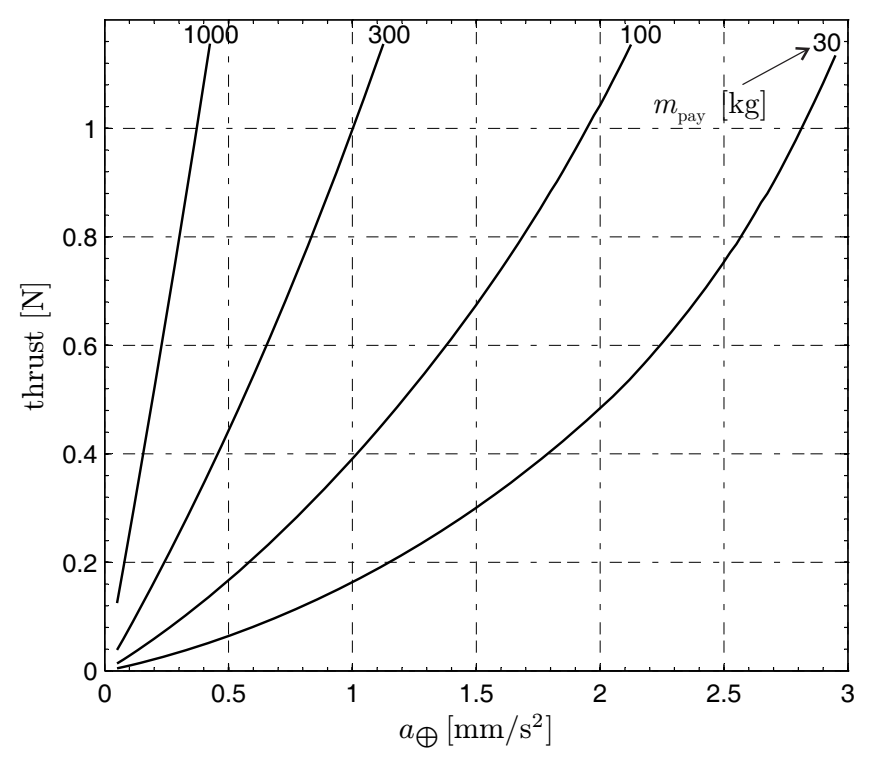

Fig. 7. E-sail thrust at $1 \mathrm{AU}$ as a function of $a_{\oplus}$ and $m_{\text {pay }}$.

$30 \mathrm{~kg}$ payload correspond to a small, first-generation E-sail, perhaps suitable for the near-term applications. The upper limits of the level curve with $m_{\text {pay }}=30 \mathrm{~kg}$ correspond, instead, to a high-performance E-sail for an advanced mission scenario as, for instance, a flyby with outer planets or a Solar System escape. On the other side, the low $a_{\oplus}$ end of the level curve with $m_{\text {pay }}=1000 \mathrm{~kg}$ represents a case where a $2.5 \mathrm{t}$ spacecraft is moved at $0.1 \mathrm{~mm} \mathrm{~s}^{-2}$ characteristic acceleration ( $3 \mathrm{~km} \mathrm{~s}^{-1}$ of $\Delta v$ per year) by a moderate size 34 -tether Esail weighing $110 \mathrm{~kg}$ (Table 2). The latter case is consistent, for example, with an advanced exploration mission towards near-Earth asteroids, which involves an in situ resource utilization and transportation (see Lewis, 1996; Gerlach, 2005).

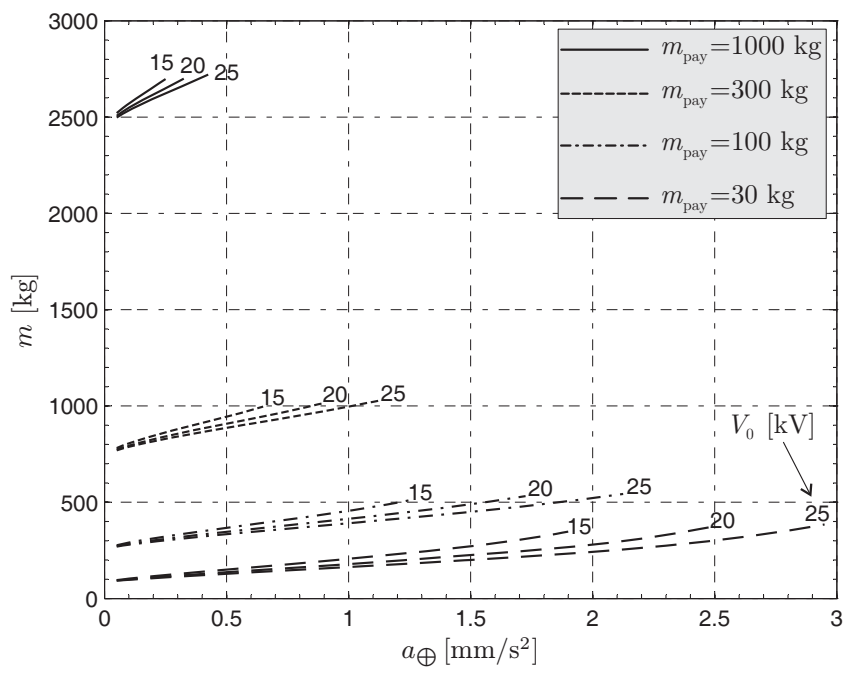

Fig. 8. Spacecraft total mass as a function of $a_{\oplus}$ and $m_{\text {pay }}$, for three values of $V_{0}: 15,20$ and $25 \mathrm{kV}$.

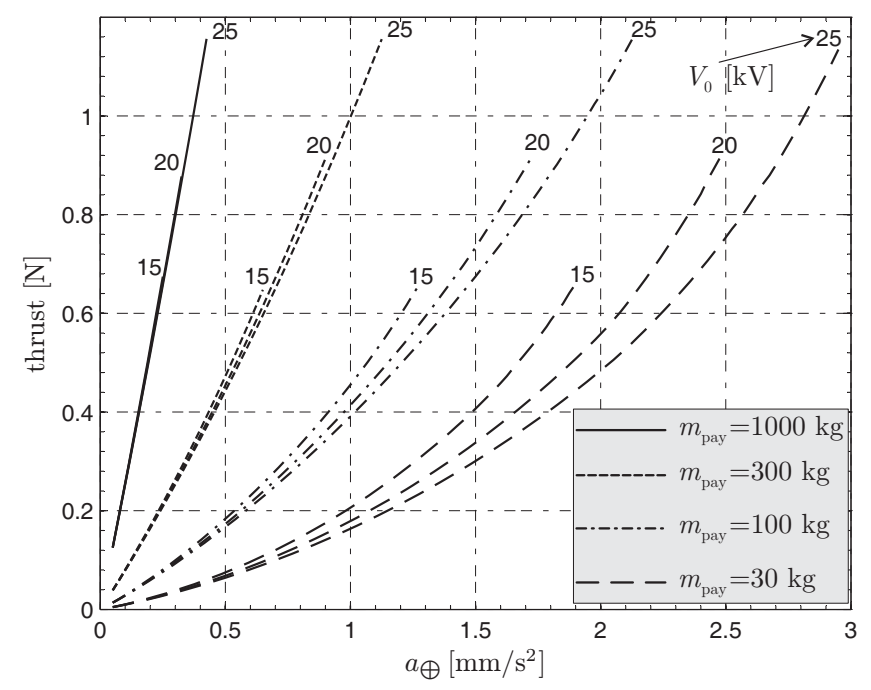

Fig. 9. E-sail thrust at $1 \mathrm{AU}$ as a function of $a_{\oplus}$ and $m_{\text {pay }}$, for three values of $V_{0}: 15,20$ and $25 \mathrm{kV}$.

\subsection{Dependence on employed voltage}

The high voltage $V_{0}$ employed at normal solar wind conditions was thus far fixed at $25 \mathrm{kV}$. We now briefly explore what happens if this value is altered. Figure 8 is similar to Fig. 6, but it shows the total mass of the spacecraft not only as a function of the characteristic acceleration $a_{\oplus}$ and payload mass $m_{\text {pay }}$, but also for three different values of $V_{0}$, namely 15,20 and $25 \mathrm{kV}$. It is seen that lowering the voltage from $25 \mathrm{kV}$ has only a minor effect on the spacecraft total mass in each case. The main effect shown in Fig. 8 is that the curves with lower $V_{0}$ end earlier. The reason is that the highest achievable characteristic acceleration depends on $V_{0}$ because the thrust per unit tether length depends on it and, by 
assumption, the maximum tether length is limited to $20 \mathrm{~km}$ while the maximum number of tethers is 100 .

Figure 9 is a generalization of Fig. 7 showing the thrust as a function of $a_{\oplus}, m_{\text {pay }}$ and $V_{0}$. Again, the effect of varying $V_{0}$ is not too significant except that the curves for lower $V_{0}$ end earlier because of the assumed total tether length limitation.

\section{Conclusions}

A detailed mathematical model has been developed for mass budget analysis and performance evaluation of an E-sail spacecraft. Our aim was to estimate the component masses as realistically as possible with current or near-term technology while including a conservative $20 \%$ overall mass margin.

Accurate mass estimates of a propulsion system are difficult to obtain, because the thruster design has usually indirect effects on other spacecraft subsystems as, for instance, the thermal and the attitude control systems. In this paper we have estimated the effective E-sail mass by evaluating the mass difference between the actual and a virtual spacecraft. The latter has the same functional components and satisfies the same environmental requirements of the former, but lacks propulsive capabilities. In that way, the indirect mass contributions are included in the estimation.

Numerical results show that the E-sail propulsion system, once qualified for flight, could be an interesting option for a wide class of deep space missions that include payloads in the range $30-1000 \mathrm{~kg}$, and require a characteristic acceleration up to about $3 \mathrm{~m} \mathrm{~s}^{-2}$. Moreover, as is shown in Table 5, some rather straightforward near-term component level improvements have the potential of reducing the effective E-sail mass further ( $28 \%$ in the specific case) with a consequent improvement in mission performance. Future work will concentrate on prototyping and testing the E-sail subsystem as well as measuring the E-sail performance on a small scale in the real environment, that is, within the solar wind.

Acknowledgements. This research was financed within the European Community's Seventh Framework Programme ([FP7/20072013]) under grant agreement number 262733 and the Academy of Finland grant decision 250591.

Edited by: H. Svedhem

\section{References}

Gerlach, C. L.: Profitably exploiting near-Earth object resources, International Space Development Conference, Washington DC, 2005, available at: http://abundantplanet.org/files/ Space-Ast-Profitably-Exploiting-NEO-Gerlach-2005.pdf (last access: 14 March 2012), 2005.
Hoyt, R. and Forward, R.: Alternate interconnection hoytether failure resistant multiline tether, US Pat. 6286788, filed: 8 September, 2000 .

Janhunen, P.: Electric sail for producing spacecraft propulsion, US Pat. 7641151, accepted 2010, filed: 2 March, 2006.

Janhunen, P.: Increased electric sail thrust through removal of trapped shielding electrons by orbit chaotisation due to spacecraft body, Ann. Geophys., 27, 3089-3100, doi:10.5194/angeo27-3089-2009, 2009.

Janhunen, P.: Photonic spin control for solar wind electric sail, Acta Astronautica, 83, 85-90, 2013.

Janhunen, P., Toivanen, P. K., Polkko, J., Merikallio, S., Salminen, P., Haeggstrom, E., Seppänen, H., Kurppa, R., Ukkonen, J., Kiprich, S., Thornell, G., Kratz, H., Richter, L., Krömer, O., Rosta, R., Noorma, M., Envall, J., Lätt, S., Mengali, G., Quarta, A. A., Koivisto, H., Tarvainen, O., Kalvas, T., Kauppinen, J., Nuottajärvi, A., and Obraztsov, A.: Electric solar wind sail: Towards test missions, Rev. Sci. Instrum., 81, 111301-1-11130111, doi:10.1063/1.3514548, 2010.

Kurppa, R., Ukkonen, J., Kiprich, S., Seppänen, H., Janhunen, P., and Haeggström, E.: Method for electric solar wind sail tether production, European Planetary Science Congress, Paper EPSC 2010-294, Rome, Italy, 19-24 September, 2010.

Larson, W. J. and Wertz, J. R.: Space mission analysis and design, 3rd Edn., Kluwer, ISBN:1-881-88310-8, 1999.

Lewis, J. S.: Mining the sky: Untold riches from the asteroid, comets, and planets, Addison-Wesley, ISBN:0-201-32819-4, 1996.

Marcuccio, S., Giusti, N., and Pergola, P.: Development of a miniaturized electric propulsion system for the E-sail project, 62nd International Astronautical Congress, Paper IAC-11.B4.6A.3, 2011.

McInnes, C. R.: Solar sailing: Technology, dynamics and mission applications, 46-54, Springer-Verlag, ISBN:3-540-21062-8, 1999.

Mengali, G., Quarta, A. A., and Janhunen, P.: Electric sail performance analysis, J. Spacecraft Rockets, 45, 122-129, doi:10.2514/1.31769, 2008.

Näsilä, A., Hakkarainen, A., Kestilä, A., Nordling, K., Modrzewski, R., Praks, J., Hallikainen, M., Saari, H., Antila, J., Mannila, R., Janhunen, P., and Vainio, R.: Aalto-1 - a hyperspectral Earth observing nanosatellite, SPIE Remote Sensing, Prague, Czech Republic, 19-22 September 2011, doi:10.1117/12.898125, 2011.

Nemzek, R. J. and Winckler, J. R.: Electron beam sounding rocket experiments for probing the distant magnetosphere, Phys. Rev. Lett., 67, 987-990, doi:10.1103/PhysRevLett.67.987, 1991.

Pajusalu, M., Rantsus, R., Pelakauskas, M., Leitu, A., Ilbis, E., Kalde, J., Lillmaa, H., Reinumägi, R., Voormansik, K., Zālite, K., Allik, V., Noorma, M., and Lätt, S.: Design of the electric power system for the ESTCube-1 satellite, Latv. J. Phys. Tech. Sci., 3, 16-24, doi:10.2478/v10047-012-0014-4, 2012.

Pappa, R. S., Blandino, J. R., Caldwell, D. W., Carroll, J. A., Jenkins, C. H. M., and Pollock, T. C.: Optical diagnostic system for solar sails: Phase 1 final report, NASA/TM-2004-213511, 2004.

Praks, J., Kestilä, A., Hallikainen, M., Saari, H., Antila, J., Janhunen, P., and Vainio, R.: Aalto-1 - an experimental nanosatellite for hyperspectral remote sensing, Geoscience and Remote Sensing Symposium (IGARSS), IEEE International, Vancouver, Canada, 25-29 July 2011, doi:10.1109/IGARSS.2011.6050199, 
2011.

Quarta, A. A. and Mengali, G.: Electric sail mission analysis for outer Solar System exploration, J. Guid. Control Dynam., 33, 740-755, doi:10.2514/1.47006, 2010.

Quarta, A. A., Mengali, G., and Janhunen, P.: Optimal interplanetary rendezvous combining electric sail and high thrust propulsion system, Acta Astronaut., 68, 603-621, doi:10.1016/j.actaastro.2010.01.024, 2011.

Seppänen, H., Kiprich, S., Kurppa, R., Janhunen, P., and Haeggström, E.: Wire-to-wire bonding of $\mu \mathrm{m}$-diameter aluminum wires for the Electric Solar Wind Sail, Microelectron. Eng., 88, 3267-3269, doi:10.1016/j.mee.2011.07.002, 2011.

Toivanen, P. K. and Janhunen, P.: Electric sailing under observed solar wind conditions, Astrophys. Space Sci. Trans., 5, 61-69, doi:10.5194/astra-5-61-2009, 2009.
Toivanen, P. K. and Janhunen, P.: Spin plane control and thrust vectoring of electric solar wind sail by tether potential modulation, J. Propul. Power, 29, 178-185, doi:10.2514/1.B34330, 2013.

Wagner, S., Sundqvist, J., and Thornell, G.: Design description of the Remote Unit, ESAIL EU FP7 project deliverable D41.2, available at: http://www.electric-sailing.fi/fp7/docs/ D412.pdf, 2012.

Wright, J. L.: Space sailing, 223-226, Gordon and Breach Science Publisher, ISBN:2-881-24842-X, 1992.

Zavyalov, M. A., Tujrujkanov, P. M., and Evlanov, E. N.: Solar ion sail electron gun study, Parts 1-2, Internal contract report of IKI/Moscow to FMI/Helsinki, 2006. 